\title{
Impacto del confinamiento por la COVID-19 en España y República Dominicana. Un estudio transcultural con jóvenes emergentes y adultos.
}

\section{Impact of Lockdown due to COVID-19 in Spain and the Dominican Republic. Transcultural Study with Emerging Adults and Adults.}

Fecha de recepción: 25-01-2021

Fecha de aceptación: 05-07-2021
Pilar Gómez-Mir

UNED-Universidad Nacional de Educación a Distancia. España

Marta Gómez-Gómez

Psytel y Arela Logopedia. España

Blas Valenzuela Contreras

Hospital General de la Plaza de la Salud Santo Domingo. República Dominicana.

\section{resumen/ahstract:}

El confinamiento debido a la COVID-19 ha supuesto un impacto mundial. El objetivo del presente estudio es analizar este impacto en adultos emergentes (AE) y población adulta residente en España y República Dominicana (RD) y las diferencias entre ambos países. Se utilizó una muestra incidental de 685 personas. Una encuesta ad hoc midió variables sociodemográficas, confianza en el sistema sanitario, miedo a la COVID-19, estrés agudo, rasgo Mindful, capacidades, dificultades, y resiliencia. Se encontraron diferencias significativas por sexo en estrés agudo, dificultades emocionales y miedo a la COVID-19 ( $p<.01)$ mayor en mujeres que en varones. En cuanto al país de residencia, presentaron mayor miedo a la enfermedad y menor confianza en el sistema sanitario público en $\mathrm{RD}$ ( $p<.01$ ). Los adultos presentaron más miedo a la enfermedad ( $p<.05)$, mayor rasgo Mindful, más conductas prosociales y mayor confianza en el sistema sanitario que los $A E(p<.01)$. Los AE puntuaron mayor estrés agudo y dificultades emocionales $(p<.01)$. Se realizó un análisis de regresión lineal, el miedo a la COVID-19 fue el mejor predictor del estrés agudo. Los resultados sugieren la necesidad de investigar factores de riesgo y protección en situaciones de pandemia valorando el país de residencia y la edad para elaborar programas de prevención eficaces.

Confinement due to COVID-19 has had a worldwide impact. The aim of this study is to analyze psychological variables involved during lockdown of emerging adults (EA) and the adult population, and the differences between Spain and the Dominican Republic (DR). A convenience sample of 685 people was used. An ad hoc online survey measured sociodemographic variables, trust in the healthcare system, fear of COVID-19, acute stress, mindful trait, capabilities, difficulties and resilience. Significant differences by sex were found in acute stress and emotional difficulties and fear of COVID-19 (p<.01) greater in women than in men. Regarding the country of residence, they presented greater fear of disease and less confidence in the public health system in the DR ( $p<.01)$. Adults showed more fear of the disease ( $<<.05)$, a greater mindful trait and enhenced prosocial behaviors (p<.01). EA scored higher acute stress and emotional difficulties (p<.01). Linear regression analysis was performed, fear of COVID-19 was the best predictor of acute stress. In conclusion, the results suggest the need for more information on differences by both nationality and age in the impact of unique stressors such as COVID-19 in order to create effective prevention programs.

\section{palabras clave/keywords:}

COVID-19; Impacto psicológico; Edad adulta emergente; Confinamiento.

COVID-19; Psychological Impact; Emerging Adults; Lockdown. 


\section{Introducción}

Desde la aparición de la COVID-19 en diciembre de 2019 se han visto afectados millones de personas. La pandemia ha supuesto un gran impacto físico, económico, social y psicológico en la población (Cao et al., 2020; Mamun y Griffiths 2020; Schimmenti et al., 2020; Wang et al., 2020). En tan sólo las primeras semanas de la aparición de la COVID-19, el número de muertes había excedido aquellas registradas por otras pandemias como el Síndrome Respiratorio Agudo Severo (SARS) (Hawryluck et al., 2004). En 2009, estudios realizados tras el brote de Influenza (H1N1) indican que entre $10 \%$ y $30 \%$ de las personas mostraron preocupación por la posibilidad de contraer el virus (Rubin et al., 2010). En el caso de los brotes del ébola con el cierre de escuelas y negocios se intensificaron las emociones desagradables de los individuos (Van Bortel et al., 2016). Esto demuestra la necesidad de valorar la salud mental de la población (Xiang et al., 2020).

El efecto causado por la pandemia y las medidas de confinamiento utilizadas con el fin de contener la enfermedad, supone que la COVID-19 podría considerarse un estresor único (Lazarus y Folkman, 1984), por las reacciones emocionales y conductuales de miedo y estrés, así como el impacto generado en la población.

Desde el inicio de la pandemia, se han realizado diversas investigaciones con el fin de evaluar las consecuencias psicológicas en diferentes poblaciones. La mayoría de los estudios concluyen que ser mujer supone un factor de riesgo, presentando así, un mayor impacto psicológico. De manera específica, las mujeres han reportado mayor sintomatología ansiosa y depresiva durante el confinamiento por COVID-19 que los hombres (Duan et al., 2020; Wang et al., 2020; Zhou et al., 2020). Sin embargo, en el estudio de Fernández Abascal, y Martín Díaz (2020) se informa de una disminución del bienestar psicológico mayor en varones que en mujeres durante el período de confinamiento, no obstante, no hubo disminución progresiva del bienestar psicológico en ambos sexos a lo largo del tiempo.

Otra variable estudiada, considerada predictora de sintomatología emocional, ha sido el estrés. El estrés tiene un efecto predictivo sobre problemas psicológicos, optimismo e inflexibilidad cognitiva en adultos. Estas dos últimas son variables que median el efecto del estrés por coronavirus (Arslan et al., 2020). Tras el primer mes de la finalización del confinamiento, un estudio con universitarios chinos, ha encontrado una prevalencia de $2.9 \%$ de estrés post traumático y un 9\% de sintomatología depresiva (Tang et al., 2020).

En relación con el afrontamiento de este evento estresante, tener la información específica de salud actualizada y medidas de precaución, se asociaron con un menor impacto psicológico (Wang et al, 2020). En estudios previos, se plantea que mostrar confianza en las recomendaciones de las autoridades sanitarias parece ser un factor protector frente al impacto psicológico de la enfermedad (Rubin et al.,2009). El uso de medidas de protección estrictas, medidas de aislamiento social, conocimiento del virus, actitud positiva (Cai et al., 2020, Blake et al., 2020; Khalid et al., 2016), apoyo social (Cai et al., 2020, Blake et al., 2020, Li et al., 2021, Malesza y Kaczmarek, 2020) y estrategias de autocuidado y promotoras de salud (Blake et al., 2020; Khalid, et al., 2016) son también estrategias reductoras de estrés ante situaciones similares. 
En cuanto a la edad, las investigaciones no son concluyentes. Por un lado, estudios como el de Wang et al. (2020) y Zhou et al. (2020) no hallaron diferencias significativas entre jóvenes y adultos, presentando así, sintomatología emocional de carácter leve en la población joven. Por otro lado, Huang y Zhao (2020) encontraron más sintomatología ansiosa y depresiva en los menores de 35 años. Kujawa et al. (2020) indican que los eventos estresantes fueron muy frecuentes entre los adultos emergentes con síntomas altos de depresión y ansiedad, aunque el estrés relacionado con la pandemia fue moderado.

La edad adulta emergente (AE) es la población que se encuentra entre los 18 y los 29 años en sociedades industrializadas (Barrera y Vinet, 2017). Se caracteriza por la inestabilidad y ser un periodo crítico donde los jóvenes están más preocupados y tensos acerca de su futuro (Côté, 2014), este periodo está descrito como un fenómeno cultural provocado por el aumento al acceso de la educación superior y la postergación de hitos como el matrimonio o acceso al trabajo más tardío que en generaciones anteriores (Arnett, 2000). Estudios acerca de este periodo evolutivo indican que, por un lado, el bienestar psicológico puede aumentar (Galambos et al., 2006) y, por otro, pueden presentar mayor vulnerabilidad frente a síntomas de ansiedad lo que puede tener un impacto negativo en su funcionamiento personal e interpersonal (Arnett, 2007). El objetivo del presente estudio es analizar el impacto provocado durante el confinamiento por la COVID-19 entre adultos emergentes y adultos mayores de 30 años provenientes de dos países distintos, España y RD. Es posible que la pandemia y las consecuencias derivadas de la misma tenga efectos a largo plazo. Por esto es importante conocer las variables relacionadas con el impacto psicosocial en estas poblaciones, por lo general poco estudiadas. Además, la valoración científica del impacto en adultos y AE permitirá realizar intervenciones y programas de prevención eficaces desde una perspectiva multidisciplinar (a nivel laboral, atención primaria, etc.), que permitan un mejor manejo ante esta situación y mitiguen las consecuencias psicológicas derivadas de la pandemia.

A continuación, y de acuerdo con la literatura previa científica en el presente estudio se pretende poner a prueba las siguientes hipótesis: (1) Los adultos emergentes reportarán un menor impacto psicológico que los adultos mayores de 30 años; (2) Las mujeres tendrán mayor sintomatología que los hombres; (3) Los residentes de RD tendrán un mayor miedo y estrés que los residentes en España; (4) En RD mostrarán menor confianza en el sistema sanitario que en España ; (5) El Miedo a la COVID-19 influirá en el Estrés agudo.

\section{Método}

\section{Participantes}

Se utilizó una muestra incidental de 685 personas $(M=35.04$; $S D=14.40)$. El 49\% son adultos (30-80 años) y el 51\% adultos emergentes (18-29 años). En cuanto al sexo, 24\% son varones y el 76\% mujeres. El 39\% residen en República Dominicana y el 61\% en España.

\section{Instrumentos}

Los datos sociodemográficos se recogieron mediante un Cuestionario ad hoc en línea. 
Escala Miedo al COVID-19 (FCV-19S; Ahorsu, et al.,2020; adaptación española de Piqueras et al., en Prensa). Escala de siete ítems con respuestas en una escala Likert de 5 puntos de 1 (muy en desacuerdo) a 5 (muy de acuerdo). La puntuación total oscila entre 7 y 35, y las puntuaciones más altas indican un mayor nivel de temor a la COVID- 19. Las propiedades psicométricas del instrumento son aceptables $(\alpha=.86, \omega=.86, \lambda 6=.86)$.

Escala del Impacto de Eventos Estresantes Revisada (IES-R; Weiss, 1996; adaptación española de Baguena et al., 2001). Consta de 22 ítems con formato de respuesta basada en una escala tipo Likert de cuatro puntos $(0=$ no del todo, $1=$ raro, $3=$ a veces, $4=$ a menudo $)$ compuesta por tres subescalas: evitación, intrusión e hiperactivación. La IES evalúa la angustia subjetiva resultante de un evento traumático de la vida. Las propiedades psicométricas son buenas, su Alpha de Cronbach oscila entre .82 y .92 para las subescalas y el total.

Cuestionario de Capacidades y Dificultades (SDQ; Bourdon y Goodman, 2005; adaptación española de Rodríguez-Hernández et al., 2012). Consta de 25 ítems con 3 opciones de respuesta $(0=$ no es cierto, $1=$ un tanto cierto, $2=$ totalmente cierto $)$, es un cuestionario de cribado clínico y evalúa 5 subescalas: hiperactividad, síntomas emocionales, problemas de comportamiento, problemas de relaciones con iguales y conducta prosocial. La consistencia interna es aceptable $(\alpha=.77)$. Se utilizó la versión de autoinforme.

Brief resilience scale (BRS; Smith et al., 2008; adaptación española de Rodríguez-Rey et al., 2016). Es una escala breve que evalúa la capacidad para resistir ante situaciones adversas o estresantes. Consta de 6 ítems con cinco opciones de respuesta ( $1=$ totalmente en desacuerdo a $5=$ totalmente de acuerdo). Una puntuación más alta indica un mayor grado de resiliencia. Su consistencia interna es adecuada $(\alpha=.83)$.

Five Facet Mindfulness Questionnaire Revised (FFMQ-R; Baer et al., 2006; adaptación española de Coo y Salanova, 2016) La escala evalúa la capacidad del sujeto para atender al presente, consta de 19 ítems y evalúa cinco subescalas: observar, describir, actuar conscientemente, no juzgar la experiencia interna y no reaccionar a la experiencia interna. La consistencia interna es aceptable, el Alfa de Cronbach es de .84.

\section{Procedimiento}

Los datos se obtuvieron durante el periodo de confinamiento en ambos países mediante una encuesta en línea difundida a través de redes sociales. Se ha realizado un diseño cuasi experimental, descriptivo y correlacional. Previamente a la recogida de datos, se obtuvo el consentimiento informado electrónicamente. Se llevó a cabo un muestreo de conveniencia donde un total de 727 personas dieron voluntariamente su consentimiento para participar en la investigación. Para este estudio se incluyeron 685 casos que cumplían los criterios de inclusión: i) ser residentes en España o RD; ii) tener entre 18 y 80 años; y iii) ser capaces de entender el español escrito.

\section{Análisis estadístico}

El análisis de los datos se realizó con el programa estadístico SPSS (versión 25). Se comprueba que la muestra no tiene una distribución normal mediante la prueba de contraste Kolmogorov-Smirnov, por lo que se utilizó la prueba no paramétrica de U Mann-Whitney 
para identificar las diferencias entre las medias de las puntuaciones de las poblaciones evaluadas y la correlación Rho de Spearman $\left(\mathrm{r}^{\mathrm{sy}}\right)$ para analizar la asociación entre las distintas variables. Se empleó la $r$ de Rosenthal para calcular el tamaño del efecto de las diferencias

Tabla 1.- Prueba de U de Mann-Whitney sobre el Estrés Agudo (IES-R), Miedo a la COVID-19 (FCV-19S), Capacidades y dificultades (SDQ) y Rasgo mindful (FFMQ-R) en función de los grupos de edad $(\mathrm{N}=685)$.

\begin{tabular}{|c|c|c|c|c|c|c|c|}
\hline Variable & Grupo & $\mathrm{N}$ & Rango promedio & $U$ & $Z$ & Sig. & $r$ \\
\hline \multicolumn{8}{|c|}{ IES-R (Estrés agudo) } \\
\hline \multirow{2}{*}{ IES-R Total } & $\mathrm{AE}$ & 347 & 366.49 & 50172.0 & \multirow{2}{*}{-3.214} & \multirow{2}{*}{.001} & \multirow{2}{*}{-.12} \\
\hline & Adultos & 338 & 317.94 & & & & \\
\hline \multirow{2}{*}{ Evitación } & $\mathrm{AE}$ & 347 & 370.14 & \multirow{2}{*}{49225.0} & \multirow{2}{*}{-3.642} & \multirow{2}{*}{.00} & \multirow{2}{*}{-.13} \\
\hline & Adultos & 338 & 315.14 & & & & \\
\hline \multirow{2}{*}{ Activación } & $\mathrm{AE}$ & 347 & 373.98 & \multirow{2}{*}{47581.0} & \multirow{2}{*}{-4.226} & \multirow{2}{*}{.00} & \multirow{2}{*}{-.16} \\
\hline & Adultos & 338 & 310.27 & & & & \\
\hline \multicolumn{8}{|c|}{ FCV-19S (Miedo a la COVID-19) } \\
\hline \multirow{2}{*}{$\begin{array}{l}\text { Miedo al } \\
\text { COVID-19 }\end{array}$} & $\mathrm{AE}$ & 347 & 327.86 & \multirow{2}{*}{53390.0} & \multirow{2}{*}{-2.032} & \multirow{2}{*}{.042} & \multirow{2}{*}{-.07} \\
\hline & Adultos & 338 & 358.54 & & & & \\
\hline \multicolumn{8}{|c|}{ SDQ (Capacidades y dificultades) } \\
\hline \multirow{2}{*}{ SDQ Total } & $\mathrm{AE}$ & 347 & 376.83 & \multirow{2}{*}{46903.50} & \multirow{2}{*}{-4.541} & \multirow{2}{*}{.00} & \multirow{2}{*}{-.17} \\
\hline & Adultos & 338 & 308.27 & & & & \\
\hline \multirow{2}{*}{ Hiperactividad } & $\mathrm{AE}$ & 347 & 377.51 & \multirow[b]{2}{*}{46668.00} & \multirow[b]{2}{*}{-4.670} & & \\
\hline & Adultos & 338 & 307.57 & & & .00 & -.17 \\
\hline Conducta & $\mathrm{AE}$ & 347 & 316.29 & & & & \\
\hline prosocial & Adultos & 338 & 370.42 & $493 / 4.50$ & -3.694 & .00 & -.14 \\
\hline Problemas & $\mathrm{AE}$ & 347 & 378.52 & & & & \\
\hline emocionales & Adultos & 338 & 306.54 & 46318.00 & -4.796 & .00 & -.18 \\
\hline Relaciones & $\mathrm{AE}$ & 347 & 373.98 & & & & \\
\hline interpersonales & Adultos & 338 & 311.20 & $4 / 894.00$ & -4.231 & .00 & -.10 \\
\hline FFMQ-R (Rasg & ndful) & & & & & & \\
\hline & $\mathrm{AE}$ & 347 & 299.64 & & & & \\
\hline FFMQ-R Total & Adultos & 338 & 387.51 & 43597.50 & -5.814 & .00 & -.22 \\
\hline Actuar & $\mathrm{AE}$ & 347 & 294.39 & 4177650 & 6534 & 00 & 24 \\
\hline consciente & Adultos & 338 & 392.90 & $41 / / 0.50$ & -0.534 & . .00 & -.24 \\
\hline No reactivo & $\mathrm{AE}$ & 347 & 326.17 & 5280200 & & 02 & -09 \\
\hline No reactivo & Adultos & 338 & 360.28 & 32802.00 & -2.200 & .02 & - .09 \\
\hline No iuzarar & $\mathrm{AE}$ & 347 & 316.58 & 4947500 & -3576 & 00 & -13 \\
\hline No juzgar & Adultos & 338 & 370.12 & $494 / 5.00$ & $-3.5 / 6$ & .00 & -.13 \\
\hline
\end{tabular}


que resultaron estadísticamente significativas. Debido a los resultados obtenidos en investigaciones previas, se llevó a cabo adicionalmente, un análisis de regresión lineal con el fin de comprobar la relación entre miedo al COVID-19 y Estrés Agudo

\section{Resultados}

Se formaron grupos en función de la edad, adultos emergentes de 18 a 29 años $(M=23.33$; $S D=2.88)$ y adultos de 30 a 77 años $(M=47.06 ; S D=11.25)$.

En función de la edad, se encontraron diferencias en las siguientes variables. Los adultos presentaron más miedo a la enfermedad $(Z=-2.032 ; p<.05)$, un mayor rasgo mindful $(Z=$

Tabla 2.- Prueba de U de Mann-Whitney sobre el Estrés Agudo (IES-R), Miedo a la COVID-19 (FCV-19S), Capacidades y dificultades (SDQ) y Rasgo mindful (FFMQ-R) en función del sexo $(\mathrm{N}=685)$.

\begin{tabular}{|c|c|c|c|c|c|c|c|}
\hline Variable & Grupo & $\mathrm{N}$ & Rango promedio & $U$ & $Z$ & Sig. & $r$ \\
\hline \multicolumn{8}{|c|}{ IES-R (Estrés agudo) } \\
\hline \multirow{2}{*}{ IES-R Total } & Mujer & 521 & 367.80 & \multirow[t]{2}{*}{29481.50} & \multirow{2}{*}{-5.965} & \multirow{2}{*}{.00} & \multirow{2}{*}{-.22} \\
\hline & Varón & 164 & 262.27 & & & & \\
\hline \multirow{2}{*}{ Evitación } & Mujer & 521 & 366.28 & \multirow{2}{*}{30594.50} & \multirow{2}{*}{-5.495} & \multirow{2}{*}{.00} & \multirow{2}{*}{-.20} \\
\hline & Varón & 164 & 269.05 & & & & \\
\hline \multirow{2}{*}{ Intrusión } & Mujer & 521 & 365.53 & \multirow{2}{*}{30984.00} & \multirow{2}{*}{-5.432} & \multirow{2}{*}{.00} & \multirow{2}{*}{-.20} \\
\hline & Varón & 164 & 271.43 & & & & \\
\hline \multirow{2}{*}{ Activación } & Mujer & 521 & 362.48 & \multirow{2}{*}{32251.50} & \multirow{2}{*}{-4.719} & \multirow{2}{*}{.00} & \multirow{2}{*}{-.18} \\
\hline & Varón & 164 & 279.16 & & & & \\
\hline \multicolumn{8}{|c|}{ FCV-19S (Miedo a la COVID-19) } \\
\hline \multirow{2}{*}{$\begin{array}{l}\text { Miedo al } \\
\text { COVID-19 }\end{array}$} & Mujer & 521 & 362.58 & \multirow{2}{*}{32522.50} & \multirow{2}{*}{-4.623} & \multirow{2}{*}{.00} & \multirow{2}{*}{-.17} \\
\hline & Varón & 164 & 280.81 & & & & \\
\hline \multicolumn{8}{|c|}{ SDQ (Capacidades y dificultades) } \\
\hline \multirow{2}{*}{ SDQ Total } & Mujer & 521 & 359.71 & \multirow{2}{*}{34013.50} & \multirow{2}{*}{-3.947} & \multirow{2}{*}{.00} & \multirow{2}{*}{-.15} \\
\hline & Varón & 164 & 289.90 & & & & \\
\hline \multirow{2}{*}{$\begin{array}{l}\text { Problemas } \\
\text { emocionales }\end{array}$} & Mujer & 521 & 366.54 & \multirow{2}{*}{30458.50} & 5501 & 00 & 21 \\
\hline & Varón & 164 & 268.22 & & -5.091 & .00 & -.21 \\
\hline FFMQ-R ( Ra & ndful) & & & & & & \\
\hline Actuar & Mujer & 521 & 323.83 & 327320 & 534 & 00 & 17 \\
\hline consciente & Varón & 164 & 403.91 & 32152.0 & ד דנ. & .00 & .17 \\
\hline
\end{tabular}


Tabla 3.- Resumen de la prueba de U de Mann-Whitney de las variables Miedo a la COVID-19 (FCV-19S), Resiliencia (BRS) y confianza en el sistema sanitario en función del lugar de residencia $(\mathrm{N}=685)$.

\begin{tabular}{|c|c|c|c|c|c|c|c|}
\hline Variable & Grupo & $\mathrm{N}$ & Rango promedio & $\mathrm{U}$ & $\mathrm{Z}$ & Sig. & $\mathrm{r}$ \\
\hline \multicolumn{8}{|c|}{ FCV-19S (Miedo a la COVID-19) } \\
\hline \multirow{2}{*}{$\begin{array}{l}\text { Miedo al } \\
\text { COVID-19 }\end{array}$} & España & 418 & 321.39 & \multirow{2}{*}{46865.50} & \multirow{2}{*}{-3.498} & \multirow{2}{*}{.00} & \multirow{2}{*}{-.13} \\
\hline & $\mathrm{RD}$ & 267 & 385.47 & & & & \\
\hline \multicolumn{8}{|c|}{ BRS (Resiliencia) } \\
\hline \multirow{2}{*}{ BRS Total } & España & 418 & 321.27 & \multirow{2}{*}{46816.50} & \multirow{2}{*}{-3.547} & \multirow{2}{*}{.00} & \multirow{2}{*}{-.13} \\
\hline & $\mathrm{RD}$ & 267 & 375.66 & & & & \\
\hline \multicolumn{8}{|c|}{ Confianza en el sistema sanitario (Cuestionario creado Ad hoc) } \\
\hline \multirow{2}{*}{ Instituciones } & España & 418 & 426.05 & \multirow{2}{*}{20830.00} & \multirow{2}{*}{-14.22} & \multirow{2}{*}{.00} & \multirow{2}{*}{-.54} \\
\hline & $\mathrm{RD}$ & 267 & 212.01 & & & & \\
\hline \multirow{2}{*}{ Personal } & España & 418 & 439.48 & \multirow{2}{*}{15230.00} & \multirow{2}{*}{-16.94} & \multirow{2}{*}{.00} & \multirow{2}{*}{-.64} \\
\hline & $\mathrm{RD}$ & 267 & 191.04 & & & & \\
\hline \multirow{2}{*}{ Instalaciones } & España & 418 & 442.42 & \multirow{2}{*}{14001.00} & \multirow{2}{*}{-16.98} & \multirow{2}{*}{.00} & \multirow{2}{*}{-.64} \\
\hline & $\mathrm{RD}$ & 267 & 186.44 & & & & \\
\hline \multirow{2}{*}{ Recursos } & España & 418 & 408.21 & \multirow{2}{*}{28268.00} & \multirow{2}{*}{-11.19} & \multirow{2}{*}{.00} & \multirow{2}{*}{-.42} \\
\hline & RD & 267 & 239.87 & & & & \\
\hline
\end{tabular}

Tabla 4.- Correlación de Spearman entre el Miedo a la COVID-19 (FCV-19S) y el Estrés agudo $($ IES-R) $(\mathrm{N}=685)$

\begin{tabular}{cccccc}
\hline & IES Total & Evitación & Intrusión & Hiperactivación \\
Miedo a la & $\begin{array}{c}\text { Coeficiente de } \\
\text { correlación }\end{array}$ & $.619^{* *}$ & $.538^{* *}$ & $.630^{* *}$ & $.505^{* *}$ \\
\hline
\end{tabular}

** La correlación es significativa en el nivel 0,01 (bilateral).

$-5.814 ; p<.01)$ y más conductas prosociales $(Z=-3.64 ; p<.01)$. Por otro lado, la población de adultos emergentes presentó puntuaciones más elevadas en las variables estrés agudo ( $Z$ $=-3.214 ; p<.01)$ y dificultades emocionales tanto en el total de la escala $(Z=-4.541 ; p<$ $.01)$, como en las subescalas: hiperactividad, problemas emocionales y relaciones interpersonales. En todas las variables el tamaño del efecto fue pequeño o muy pequeño $(r<.20)$ (Tabla 1). 
Tabla 5.- Resumen de las puntuaciones de las regresiones lineales sobre el Miedo a la COVID-19 (FCV-19S)

\begin{tabular}{lcccc}
\hline & $\mathrm{F}(\mathrm{p})$ & $\mathrm{R} 2$ ajustada & $\mathrm{B}$ & $\mathrm{t}(\mathrm{p})$ \\
\hline M1: Predictor (Regresión lineal simple) & & & \\
Estrés agudo (IES-R) & $271.365^{* *}$ & .405 & 1.767 & $16.473^{* *}$ \\
\hline
\end{tabular}

Variable dependiente: Miedo a la COVID-19 (FCV-19S)

Variable independiente: Modelo 1: Estrés agudo (IES-R).

Nota: $* * p<, 01$

Se encontraron diferencias significativas por sexo en estrés agudo $(Z=-5.965 ; p<.01)$, dificultades emocionales $(Z=-5.591 ; p<.01)$ y miedo a la COVID-19 $(Z=-4.623 ; p<$ .01). Las mujeres puntuaron más alto que los varones en las anteriores escalas. Con respecto al rasgo mindful se hallaron diferencias significativas en la subescala Actuar Consciente, presentando puntuaciones más elevadas los varones $(Z=-4.531 ; p<.01)$. En todas las variables el tamaño del efecto fue pequeño o muy pequeño $(r<.20)$, (véase Tabla 2 )

En cuanto al país de residencia, presentaron mayor miedo a la enfermedad los residentes en $\operatorname{RD}(Z=-3.498 ; p<.01)$ así como puntuaciones más elevadas en resiliencia $(Z=-3.547 ; p$ $<.01)$. Asimismo, se obtuvieron diferencias significativas entre residencia y confianza en el sistema sanitario público en todos los ítems: instituciones $(Z=-14.22 ; p<.01)$, personal $(Z=-16.94 ; p<.01)$, instalaciones $(Z=-16.98 ; p<.01)$ y recursos $(Z=-11.19 ; p<.01)$, presentando una mayor confianza los residentes españoles. En todos los casos el tamaño del efecto fue medio o grande, los valores de $r$ oscilaron entre - .42 y -.64 (Tabla 3).

Adicionalmente, se llevó a cabo un análisis de predicción mediante estudios de regresión lineal entre las variables Miedo a la COVID-19 y Estrés Agudo, tras analizar que entre ambas variables existe una correlación directa y significativa $(p<.01)$ entre ambas variables(véase Tabla 4) El modelo predijo alrededor de un $40 \%$ de la varianza explicada $\left(F=271.365, R^{2}\right.$ $=.405 ; p<.01)($ Tabla 5$)$.

\section{Discusión}

El presente estudio tiene como objetivo evaluar el impacto psicológico del confinamiento debido a la COVID-19 en una muestra de adultos y adultos emergentes residentes en España y RD y las variables implicadas en dicho impacto. La pandemia ha tenido efectos negativos para la población en todos los ámbitos (Cao et al., 2020; Huang y Zhao, 2020; Lei et al., 2020; Peng et al., 2020) y por ello es relevante identificar, por un lado, la sintomatología y dificultades psicológicas asociadas a la COVID-19 y por otro, las variables protectoras que favorecerá un mejor afrontamiento de este estresor único.

Los resultados obtenidos son acordes a la mayoría de los estudios previos. Las mujeres presentan mayor miedo al COVID-19, estrés agudo y sintomatología emocional que los varones (Zhou et al. 2020; Malesza y Kaczmarek, 2020). 
Los adultos mostraron mayor miedo, posiblemente porque parte de la población analizada se encuentra dentro de población de riesgo. Sin embargo, la edad emergente reportó más estrés y dificultades emocionales (Zhou et al., 2020; Liang et al., 2020)

En relación con la variable edad en diversas investigaciones se indica que los AE formarían parte de la población vulnerable al impacto de la pandemia. Esto puede resultar sorprendente ya que la COVID-19 es una enfermedad que supone un mayor riesgo para poblaciones de mediana y tercera edad que para los jóvenes. Una posible explicación es que los AE se encuentran en una fase del desarrollo en el que perciben una mayor incertidumbre y ansiedad por el futuro, sería interesante valorar si esta población ya presentaba niveles más elevados de ansiedad y depresión previos a la pandemia. (Germani et al., 2020).

En cuanto al lugar de residencia, los residentes en RD mostraron mayor miedo a la enfermedad, estos resultados concuerdan con estudios previos (Piqueras et al.; en Prensa; Gómez et al., 2020). Las diferencias en el sistema sanitario público podrían explicar la menor confianza en RD, en el que el sistema público de salud no cubre todas las necesidades sanitarias por igual en función de la zona en la que se resida, hay un número escaso de profesionales sanitarios y de hospitales a los que puede accederse, y la estructura de atención primaria es insuficiente. Asimismo, también se podría explicar el mayor miedo a la COVID-19 en esta población. El apoyo social y la resiliencia se ha asociado a una mejor salud mental, en los casos de baja resiliencia, el apoyo social amortigua los efectos negativos en la misma ( $\mathrm{Li}$ et al.,2021) Los resultados obtenidos indican que los residentes en RD muestran un nivel mayor de resiliencia, esto podría deberse a la exposición de esta población a otros eventos estresantes como catástrofes naturales. Sería interesante llevar a cabo un seguimiento y valorar si una mayor resiliencia, a largo plazo, disminuye los efectos psicológicos provocados por una situación como la actual.

El análisis de regresión adicional permitió identificar como el Miedo a la COVID-19 predecía en una gran proporción el Estrés agudo, datos acordes con la validación en español del FCV-19S (Piqueras et al., en Prensa; Gómez et al., 2020; Satici 2020). La literatura reciente sobre los efectos provocados por la pandemia indica que existen poblaciones más vulnerables a presentar un mayor impacto negativo. Como, por ejemplo: los adultos emergentes, las mujeres, personas con un mayor miedo a enfermar previo a la pandemia.

En relación con las investigaciones centradas en la búsqueda de intervenciones eficaces concretas para paliar este impacto, Baiano et al. (2020) sugieren que los programas seleccionados para disminuir las consecuencias de esta situación deben estar incluidos en el marco de la terapia cognitivo conductual y en particular, en intervenciones cognitivo conductuales con un enfoque basado en la atención plena ya que éstas favorecen la capacidad de centrar la atención y la conciencia en el momento presente. Otra propuesta de intervención basada en mindfulness es la de Weis et al., (2020). Estos autores aplican una intervención basada en mindfulness (Koru-Mindfulness) y tras ella, los AE presentaron mayor autocompasión, mindfulness, menor estrés, ansiedad y problemas de sueño. Además, Zheng et al., (2020) realizaron dos estudios en los que comprobaron que el mindfulness inducido amortigua el impacto de los estresores por la COVID-19 y era un eficaz neutralizador del efecto negativo 
de las consecuencias de la pandemia tanto en el sueño como en el trabajo.

Sería relevante priorizar la aplicación de estas intervenciones en dichas poblaciones en riesgo o más vulnerables y continuar con esta línea de investigación para la creación de programas específicos que favorezcan el desarrollo de estrategias de afrontamiento más adecuadas ante eventos estresantes como la COVID-19.

Este estudio tiene como limitaciones el haber utilizado una muestra incidental y una mayor representación de mujeres, así cómo utilizar instrumentos no validados específicamente en población dominicana a excepción del FCV-19S (Piqueras et al., en Prensa). Sin embargo, hay pocos estudios transculturales que valoren el impacto psicológico del confinamiento en estas poblaciones y que evalúen el rasgo mindful como variable de afrontamiento ante una situación estresante.

Los efectos psicológicos a largo plazo son aún desconocidos. Las medidas de confinamiento, el distanciamiento social y las dificultades económicas asociadas a la pandemia no van a desaparecer de forma inmediata. El deterioro de la salud mental de poblaciones específicas como los sanitarios o la tercera edad son en este momento una realidad. Por tanto, es importante continuar profundizando en el conocimiento de variables moduladoras que minimicen dicho impacto y promover la creación e implementación de programas de prevención dirigidos a todas las edades y en diferentes ámbitos, que favorezcan una mejor adaptación a esta nueva realidad.

\section{Referencias}

Ahorsu, D. K., Lin, C.-Y., Imani, V., Saffari, M., Griffiths, M. D., \& Pakpour, A. H. (2020). The Fear of COVID-19 Scale: Development and Initial Validation. International Journal of Mental Health and Addiction. doi:10.1007/s11469-020-00270-8

Arnett, J. J. (2000). Emerging adulthood: A theory of development from the late teens through the twenties. American Psychologist, 55(5), 469. doi:10.1037//0003-066X.55.5.469

Arnett, J.J (2007). Adultez emergente: ¿qué es y para qué sirve? Perspectivas del desarrollo infantil, 1(2), 68-73

Arslan, G., Yıldırım, M., Tanhan, A., Bulu, M., \& Allen, K. A. (2020). Coronavirus stress, optimism-pessimism, psychological inflexibility, and psychological health: Psychometric properties of the Coronavirus Stress Measure. International Journal of Mental Health and Addiction, 1. doi:10.1007/s11469-020-00337-6

Baer, R. A., Smith, G. T., Hopkins, J., Krietemeyer, J., \& Toney, L. (2006). Using self-report assessment methods to explore facets of mindfulness. Assessment, 13(1), 27-45.

Baiano, C., Zappullo, I., \& Conson, M. (2020). Tendency to worry and fear of mental health during Italy's COVID-19 lockdown. International journal of environmental research and public health, 17(16), 5928. doi: 10.3390/ijerph17165928

Baguena, M.J., Villarroya, E., Beleña, A., Roldan, C., \& Reig, R. (2001). Psychometric properties of the Spanish version of the Impact of Event Scale-Revised (IES-R). Análisis y Modificación de Conducta, 271114), 581-604.

Barrera-Herrera, A., \& Vinet, E. V. (2017). Adultez Emergente y Características culturales de la etapa en Universitarios chilenos. Terapia Psicológica, 35(1), 47-56. doi:10.4067/S0718-48082017000100005

Blake, H., Bermingham, F., Johnson, G., \& Tabner, A. (2020). Mitigating the psychological impact of COVID-19 on healthcare workers: a digital learning package. International Journal of Environmental Research and Public Health, 17(9), 2997. doi:10.3390/ ijerph17092997 
Bourdon, K. H., Goodman, R., Rae, D. S., Simpson, G., \& Koretz, D. S. (2005). The Strengths and Difficulties Questionnaire: US normative data and psychometric properties. Journal of the American Academy of Child \& Adolescent Psychiatry, 44(6), 557-564. doi:10.1097/01.chi.0000159157.57075.c8

Cai, H., Tu, B., Ma, J., Chen, L., Fu, L., Jiang, Y., \& Zhuang, Q. (2020). Psychological Impact and Coping Strategies of Frontline Medical Staff in Hunan Between January and March 2020 During the Outbreak of Coronavirus Disease 2019 (COVID-19) in Hubei, China. Medical Science Monitor, 26, e924171-1. doi:10.12659/MSM.924171

Cao, W., Fang, Z., Hou, G., Han, M., Xu, X., Dong, J., \& Zheng, J. (2020). The psychological impact of the COVID-19 epidemic on college students in China. Psychiatry Research, 217, 112934. doi:10.1016/j.psychres.2020.112934

Coo, \& Salanova (2016). Validación española de la escala abreviada Five Facet Mindfulness Questionnaire (FFMQ): Un estudio piloto.

Côté, J. (2014). Youth studies: Fundamental issues and debates. Macmillan International Higher Education.

Duan, L., Shao, X., Wang, Y., Huang, Y., Miao, J., Yang, X., \& Zhu, G. (2020). An investigation of mental health status of children and adolescents in China during the outbreak of COVID-19. Journal of Affective Disorders. doi:10.1016/j.jad.2020.06.029

Fernández-Abascal, E. G., \& Martín-Díaz, M. D. (2020). Longitudinal study on affect, psychological well-being, depression, mental and physical health, prior to and during the COVID-19 pandemic in Spain. Personality and Individual Differences, 110591. doi: 10.1016/j.paid.2020.110591

Galambos, N. L., Barker, E. T., \& Krahn, H. J. (2006). Depression, self-esteem, and anger in emerging adulthood: seven-year trajectories. Developmental psychology, 42(2), 350.

Germani, A., Buratta, L., Delvecchio, E., Gizzi, G., \& Mazzeschi, C. (2020). Anxiety severity, perceived risk of COVID-19 and individual functioning in emerging adults facing the pandemic. Frontiers in psychology, 11.

Gómez, M. G., Mir, P. G., \& Valenzuela, B. (2020). Adolescencia y edad adulta emergente frente al COVID-19 en España y República Dominicana. Revista de Psicología Clínica con Niños y Adolescentes, 73), 35-41. doi: 10.21134/rpcna.2020.mon.2043

Hawryluck, L., Gold, W. L., Robinson, S., Pogorski, S., Galea, S., \& Styra, R. (2004). SARS control and psychological effects of quarantine, Toronto, Canada. Emerging Infectious Diseases, 1977), 1206. doi:10.3201/ eid1007.030703

Huang, Y., \& Zhao, N. (2020). Mental health burden for the public affected by the COVID-19 outbreak in China: Who will be the high risk group? Psychology, Health \& Medicine, 1-12. doi:10.1080/13548506.2020.1754438

Khalid, I., Khalid, T. J., Qabajah, M. R., Barnard, A. G., \& Qushmaq, I. A. (2016). Healthcare workers emotions, perceived stressors and coping strategies during a MERS-CoV outbreak. Clinical Medicine \& Research, 14(1), 7-14. doi: 10.3121/cmr.2016.1303

Kujawa, A., Green, H., Compas, B. E., Dickey, L., \& Pegg, S. (2020). Exposure to COVID-19 pandemic stress: Associations with depression and anxiety in emerging adults in the United States. Depression and anxiety, 3712), 1280-1288. doi: https://doi. org/10.1002/da.23109

Lazarus, R. S., \& Folkman, S. (1984). Stress, appraisal, and coping. Springer Publishing Company.

Liang, L., Ren, H., Cao, R., Hu, Y., Qin, Z., Li, C., \& Mei, S. (2020). The effect of COVID-19 on youth mental health. Psychiatric Quarterly, 1-12. doi:10.1007/s11126-020-09744-3

Lei, L., Huang, X., Zhang, S., Yang, J., Yang, L., \& Xu, M. (2020). Comparison of prevalence and associated factors of anxiety and depression among people affected by versus people unaffected by quarantine during the COVID-19 epidemic in Southwestern

China. Medical Science Monitor: International Medical Journal of Experimental and Clinical Research, 26, e924609-1. doi:10.12659/ MSM.924609.

Malesza, M., \& Kaczmarek, M. C. (2020). Predictors of anxiety during the COVID-19 pandemic in Poland. Personality and individual differences, 170, 110419 .

Mamun, M. A., \& Griffiths, M. D. (2020). First COVID-19 suicide case in Bangladesh due to fear of COVID-19 and xenophobia: Possible suicide prevention strategies. Asian Journal of Psychiatry, 51, 102073. doi:10.1016/j. ajp.2020.102073

Peng, L., Zhang, J., Li, M., Li, P., Zhang, Y., Zuo, X., et al. (2012). Negative life events and mental health of Chinese medical students: the effect of resilience, personality and social support. Psychiatry Research, 196(1), 138-141. doi:10.1016/j. psychres.2011.12.006

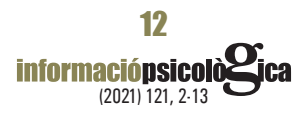


Piqueras, J. A., Gómez-Gómez, M., Marzo, J. C., Gómez-Mir, P., Falco, R. \& Valenzuela, B., The PSICO-RECURSOS COVID-19 study group (2020) Validation of the Spanish version of Fear of COVID-19 Scale: Its association with acute stress and coping (en prensa)

Rodríguez-Hernández, P. J., Betancort, M., Ramírez-Santana, G. M., García, R., Sanz-Álvarez, E. J., \& De las Cuevas-Castresana, C. (2012). Psychometric properties of the parent and teacher versions of the Strength and Difficulties Questionnaire (SDO) in a Spanish sample. International Journal of Clinical and Health Psychology, 12(2), 265-279.

Rodríguez-Rey, R., Alonso-Tapia, J., \& Hernansaiz-Garrido, H. (2016). Reliability and validity of the Brief Resilience Scale (BRS) Spanish Version. Psychological assessment, 28(5), e101.

Rubin, G. J., Amlôt, R., Page, L., \& Wessely, S. (2009). Public perceptions, anxiety, and behaviour change in relation to the swine flu outbreak: cross sectional telephone survey. The British Medical Journal.

Rubin, G. J., Potts, H. W. W., \& Michie, S. (2010). The impact of communications about swine flu (influenza A H1N1v) on public responses to the outbreak: results from 36 national telephone surveys in the UK. Health Technology Assessment, 14(34), 183-266.

Satici, B., Gocet-Tekin, E., Deniz, M. E., \& Satici, S. A. (2020). Adaptation of the Fear of COVID-19 Scale: Its association with psychological distress and life satisfaction in Turkey. International Journal of Mental Health and Addiction, 1. doi: 10.1007/ s11469-020-00294-0.

Schimmenti, A., Billieux, J., \& Starcevic, V. (2020). The four horsemen of fear: an integrated model of understanding fear experiences during the COVID-19 pandemic. Clinical Neuropsychiatry, 17(2), 41-45. doi:10.36131/ CN20200202

Smith, B. W., Dalen, J., Wiggins, K., Tooley, E., Christopher, P., \& Bernard, J. (2008). The brief resilience scale: assessing the ability to bounce back. International journal of behavioral medicine, 15(3), 194-200. doi: 10.1080/10705500802222972

Tang, W., Hu, T., Hu, B., Jin, C., Wang, G., Xie, C., \& Xu, J. (2020). Prevalence and correlates of PTSD and depressive symptoms one month after the outbreak of the COVID-19 epidemic in a sample of home-quarantined Chinese university students. Journal of Affective Disorders. doi:10.1016/j. jad.2020.05.009

Van Bortel, T., Basnayake, A., Wurie, F., Jambai, M., Koroma, A. S., Muana, A. T., ... \& Nellums, L. B. (2016). Psychosocial effects of an Ebola outbreak at individual, community and international levels. Bulletin of the World Health Organization, 94(3), 210. doi:10.2471\%2FBLT.15.158543

Wang, C., Pan, R., Wan, X., Tan, Y., Xu, L., Ho, C. S., \& Ho, R. C. (2020). Immediate psychological responses and associated factors during the initial stage of the 2019 coronavirus disease (COVID-19) epidemic among the general population in China. International Journal of Environmental Research and Public Health, 17(5), 1729. doi:10.3390/ijerph17051729

Weiss, D. S., \& Marmar, C. R. (1997). The Impact of Event Scale-Revised. In J. P. Wilson and T. Keane (Eds.), Assessing psychological trauma and PTSD: A handbook for practitioners. New York: Guilford Press.

Weis, R., Ray, S. D., \& Cohen, T. A. (2020) Mindfulness as a way to cope with COVID-19 related stress and anxiety. Counselling and Psychotherapy Research.

Weiss, D. S. (1996): Impact of Events Scale-Revised. In H. Stamm (Ed.), Measurement of stress, trauma, and adaptation (pp. 186-188). The Sidran Press.

Xiang, Y. T., Yang, Y., Li, W., Zhang, L., Zhang, O., Cheung, T., \& Ng, C. H. (2020). Timely mental health care for the 2019 novel coronavirus outbreak is urgently needed. The Lancet Psychiatry, 73), 228-229. doi:10.1016/ S2215-0366(20)30046-8

Zheng, M. X., Masters-Waage, T. C., Yao, J., Lu, Y., Tan, N., \& Narayanan, J. (2020). Stay Mindful and Carry on: Mindfulness Neutralizes COVID-19 Stressors on Work Engagement via Sleep Duration. Frontiers in Psychology, 11.

Zhou, S. J., Zhang, L. G., Wang, L. L., Guo, Z. C., Wang, J. O., Chen, J. C., \& Chen, J. X. (2020). Prevalence and socio-demographic correlates of psychological health problems in Chinese adolescents during the outbreak of COVID-19. European Child \& Adolescent Psychiatry, 1-10. doi:10.1007/s00787-020-01541-4. 